\title{
Terapia de oxígeno hiperbárico complementaria al desbridamiento en el control de la gangrena de Fournier: utilidad de la puntuación de un índice de severidad en la predicción de la gravedad de la enfermedad y la supervivencia del paciente
}

\author{
A. Janane ${ }^{a, *}$, F. Hajji ${ }^{a}$, T.O. Ismail ${ }^{a}$, J. Chafiqui ${ }^{a}$, M. Ghadouane ${ }^{a}$, \\ A. Ameur ${ }^{a}$, M. Abbar ${ }^{a}$ y A. Albouzidi ${ }^{\text {b }}$ \\ a Departamento de Urología, Hospital Militar Universitario, Facultad de Medicina Mohamed V, Rabat, Marruecos \\ b Departamento de Patología, Hospital Militar Universitario, Facultad de Medicina Mohamed V, Rabat, Marruecos
}

Recibido el 22 de noviembre de 2010; aceptado el 1 de enero de 2011

Accesible en línea el 14 de abril de 2011

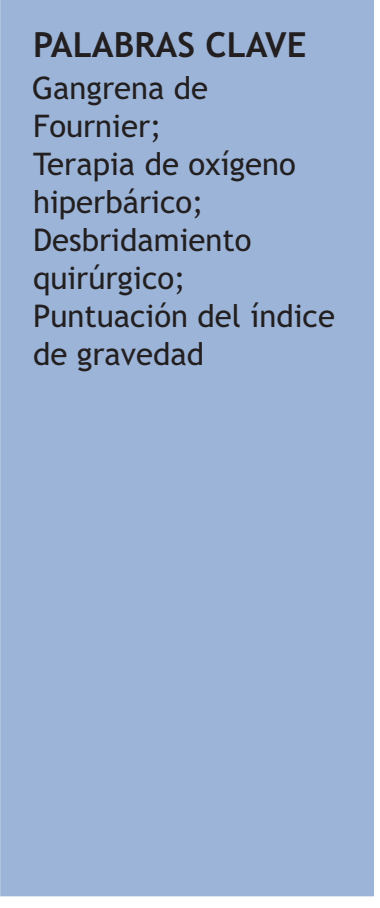

\begin{abstract}
Resumen
Introducción: La terapia de oxígeno hiperbárico (TOHB) concomitante con la cirugía ha demostrado una mejora en la reducción de la mortalidad por gangrena de Fournier (GF) en comparación con la aplicación exclusiva de desbridamiento quirúrgico. La mayoría de los datos provienen de centros con un número relativamente reducido de pacientes, y en los que se emplea solamente un procedimiento quirúrgico. El objetivo planteado consistía en evaluar la eficiencia del desbridamiento agresivo con TOHB complementaria, así como evaluar el valor predictivo del índice de puntuación de gravedad de la gangrena de Fournier (IGGF).

Material y métodos: 70 gangrenas de Fournier (GF) tratadas con desbridamiento quirúrgico y TOHB. Los datos evaluados fueron los resultados de las exploraciones físicas, los análisis de laboratorio tanto en el momento del ingreso como los finales, la extensión del desbridamiento quirúrgico y el antibiótico utilizado. Los pacientes recibieron TOHB complementaria. Se desarrolló un IGGF con el fin de adjudicar una puntuación que describiese la gravedad de la enfermedad. Este índice tiene en cuenta las constantes vitales de los pacientes, los parámetros metabólicos (niveles de sodio, potasio, creatinina y bicarbonato, así como recuento de linfocitos) y calcula una puntuación relativa a la gravedad de la enfermedad en ese momento. Se evaluaron los datos en función de la supervivencia o no del paciente. Todos los pacientes fueron sometidos a desbridamiento quirúrgico, realizándose el desbridamiento de la herida de forma periódica en el periodo postoperatorio.

Resultados: De un total de 70 pacientes fallecieron 8 (el 11,4\%) y sobrevivieron 62 (el 88,5\%). La diferencia de edad entre los supervivientes (edad media 50,0 años) y no supervivientes (edad media 54,5 años) no fue significativa ( $p=0,321)$. La extensión media del área del cuerpo afectada por el proceso de necrosis en los pacientes que sobrevivieron y en los que no
\end{abstract}

\footnotetext{
* Autor para correspondencia.

Correo electrónico: a.janane@yahoo.fr (A. Janane).
} 


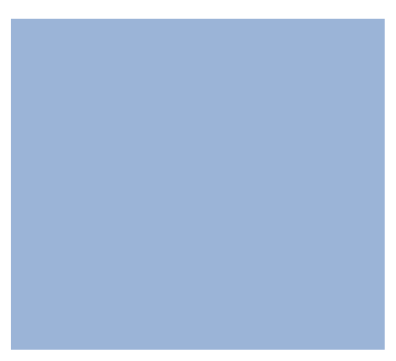

\section{KEYWORDS \\ Fournier's gangrene; Hyperbaric oxygen therapy; \\ Surgical debridement; Severity index score}

sobrevivieron era del 2,4 y del $4,9 \%$, respectivamente $(p=0,001)$. Excepto en lo referente a la albúmina, no se encontraron diferencias significativas entre supervivientes y no supervivientes. Las puntuaciones medias en el IGGF en el momento del ingreso de los supervivientes y de los no supervivientes fueron de $2,1 \pm 2,0$ y de 4,2 $\pm 3,8,(p=0,331)$.

Conclusión: La puntuación del IGGF no resultó ser un factor de predicción de la gravedad, de la enfermedad ni de la supervivencia del paciente. Sin embargo, tanto las alteraciones metabólicas como la extensión de la enfermedad aparecieron como factores significativos de riesgo en cuanto a predicción de la gravedad de la GF y la supervivencia del paciente. (c) 2010 AEU. Publicado por Elsevier España, S.L. Todos los derechos reservados.

Hyperbaric oxygen therapy adjunctive to surgical debridement in management of Fournier's gangrene: usefulness of a severity index score in predicting disease gravity and patient survival

\section{Abstract}

Introduction: Hyperbaric oxygen therapy (HBOT) concomitant to surgery has been reported to reduce Fournier's gangrene (FG) mortality compared to exclusive surgical debridement. Most report from centers with relatively few patients using only surgical procedure. To assess efficiency of aggressive debridement with adjunctive HBOT. To evaluate Fournier's gangrene severity score index (FGSI) predictive value.

Material and methods: 70 Fournier's gangrene (FG) treated by surgical debridement and HBOT. Data were evaluated physical examination findings, admission and final laboratory tests, surgical debridement extent, and antibiotic used. Patients had adjunctive (HBOT). FGSI, developed to assign a score describing the acuity of disease, was used. This index presents patients' vital signs, metabolic parameters (sodium, potassium, creatinine, and bicarbonate levels, and white blood cell count) and computes a score relating to the severity of disease at that time. Data were assessed according to whether the patient survived or died. All patients underwent surgical debridement. Wound debridement was regularly performed in the post operative period.

Results: Of 70 patients, 8 died (11.4\%) and 62 survived (88.5\%). Difference in age between survivors (median age, $50.0 \mathrm{yr}$ ) and non survivors (median age, $54.5 \mathrm{yr}$ ) was not significant $(p=0.321)$. Median extent of body surface area involved in necrotizing process in patients who survived and did not survive was $2.4 \%$ and $4.9 \%$, respectively $(p=0.001)$. Except for albumin, no significant differences were found between survivors and nonsurvivors. Median admission FGSI scores for survivors and non survivors were $2.1 \pm 2.0$ and $4.2 \pm 3.8,(p=0.331)$.

Conclusion: FGSI score did not predict disease severity and the patient's survival. Metabolic aberrations, extent of disease seemed to be important risk factors for predicting FG severity and patient survival.

(c) 2010 AEU. Published by Elsevier España, S.L. All rights reserved.

\section{Introducción}

La fascitis necrosante potencialmente mortal, que afecta a la región perineal y perianal del tracto genitourinario masculino fue descrita por primera vez por Jean-Alfred Fournier en $1883^{1}$. Si la enfermedad se extiende a la pared abdominal se puede denominar también gangrena de Meleney ${ }^{2}$. Una revisión reciente ${ }^{3}$ indica que la gangrena de Fournier (GF) suele afectar a hombres mayores, se basa en afecciones médicas predisponentes y su etiología suele poderse identificar sin dificultad en la mayoría de casos.

A pesar de que se ha avanzado en el conocimiento de la etiología, el diagnóstico, el tratamiento y las técnicas de cuidados intensivos, el índice de mortalidad de la GF sigue situándose entre el 10 y el $50 \%^{3,4}$. Laor et al fueron los primeros en describirla, creando posteriormente el índice de gravedad de la gangrena de Fournier (IGGF) para predecir la gravedad de la enfermedad ${ }^{5}$. En el presente estudio se compararon los datos fisiopatológicos medibles de supervivientes y no supervivientes a la GF con ayuda de la puntuación IGGF.
En este estudio revisamos nuestros 10 años de experiencia con 70 pacientes tratados de GF con el fin de identificar los factores etiológicos y exponer la validez del IGGF como factor de predicción de la gravedad de la enfermedad y la supervivencia de los pacientes.

\section{Pacientes y métodos}

\section{Pacientes}

Se incluyó en el estudio de manera consecutiva a 77 varones con una edad media de 63,5 años (intervalo de 35 a 76 años), pacientes a los que se trató de GF en nuestro departamento universitario entre enero de 2000 y agosto de 2009. Se recopiló información relativa a historia médica, síntomas, resultados de las exploraciones físicas y constantes vitales. El seguimiento de los pacientes se hizo mediante análisis hematológicos y bioquímicos diarios. Se tomó nota de los resultados de todos los análisis bioquímicos, hematológicos y microbiológicos en la evaluación realizada en el momento 
Tabla 1 Índice de gravedad de la gangrena de Fournier ${ }^{4}$

\begin{tabular}{|c|c|c|c|c|c|c|c|c|c|}
\hline \multirow[t]{2}{*}{ Variables } & \multicolumn{4}{|c|}{ Valores anómalos elevados } & \multirow[b]{2}{*}{0} & \multicolumn{4}{|c|}{ Valores anómalos reducidos } \\
\hline & +4 & +3 & +2 & +1 & & +1 & +2 & +3 & +4 \\
\hline Temperatura, ${ }^{\circ} \mathrm{C}$ & $>41$ & $39-40,9$ & & $38,5-35,9$ & $35-38,4$ & $34 \cdot 35,9$ & $32-33,9$ & $30-31,9$ & $<29,9$ \\
\hline Ritmo cardíaco & $>180$ & $140-179$ & $110-139$ & & $70-109$ & - & $55-69$ & $40-54$ & $<39$ \\
\hline Frecuencia respiratoria & $>50$ & $35-49$ & & $25-34$ & $12-24$ & $10-11$ & $6-9$ & & $<5$ \\
\hline Na plasmático, mmol/ l & $>180$ & $160-179$ & $155-159$ & $150-154$ & $130-149$ & & $120-129$ & $111-119$ & $<110$ \\
\hline $\mathrm{K}++$ plasmático, mmol/ l & $>7$ & $6-6,9$ & - & $5,5-5,9$ & $3,5-5,4$ & $3-3,4$ & $2,5-2,9$ & - & $<2,5$ \\
\hline $\begin{array}{l}\text { Creatinina en sangre, } \\
\text { mg/ } 100 \mathrm{ml}, \times 2 \text { en } \\
\text { insuficiencia renal } \\
\text { aguda }\end{array}$ & $>3,5$ & $2-3,4$ & $1,5-1,9$ & - & $0,6-1,4$ & - & $<0,6$ & - & - \\
\hline$\%$ de hematocrito & $>60$ & - & $50-59,9$ & $46-49,4$ & $30-45,9$ & - & $20-29,9$ & - & $<20$ \\
\hline $\begin{array}{l}\text { Recuento de glóbulos } \\
\text { blancos, total/ } \mathrm{mm}^{3} \mathrm{x} \\
1.000\end{array}$ & $>40$ & - & $20-39,9$ & $15-19,9$ & $3-14,9$ & - & $1-2,9$ & - & $<1$ \\
\hline $\begin{array}{l}\text { Bicarbonato plasmático, } \\
\mathrm{mmol} / \mathrm{l}\end{array}$ & $>52$ & $41-51,9$ & - & $32-40,9$ & $22-31,9$ & - & $18-21,9$ & $15-17,9$ & $<15$ \\
\hline
\end{tabular}

del ingreso y en la evaluación final. Los criterios para determinar la aparición de los síntomas fueron fiebre $>38^{\circ} \mathrm{C}$, eritema o inflamación del escroto, purulencia o supuración de la herida, fluctuación o crepitación.

\section{Métodos}

Se calculó la extensión de la gangrena para los monogramas del área del cuerpo modificados que suelen utilizarse en la evaluación de la extensión de las lesiones por quemadura: el pene, el escroto y el perineo representaban un $1 \%$ del cuerpo respectivamente, siendo cada fosa isquiorrectal un $2,5 \%$, etc.

Se llevaron inmediatamente todas las muestras de cultivo al laboratorio clínico de microbiología. El material se dividió en dos placas (agar eosina-azul de metileno [EMB] y agar sangre simple). Las dos placas se incubaron al aire a $37^{\circ} \mathrm{C}$ durante 24 horas para analizar los microorganismos aerobios. Se sellaron otras placas de agar EMB y de agar sangre simple en una bolsa de plástico Zip-Loc para mantener la atmósfera con incremento de $\mathrm{CO}_{2}$ a $37^{\circ} \mathrm{C}$ durante 24 horas para analizar los microorganismos anaerobios. A la espera de los resultados de los cultivos y de las pruebas de sensibilidad, se administró un doble tratamiento de antibióticos por vía parenteral con cefalosporina de tercera generación (ceftriaxona $4 \mathrm{~g} / \mathrm{d}$ ) y $1,5 \mathrm{~g} / \mathrm{d}$ de metronidazol. Durante el periodo postoperatorio se efectuaba a todos los pacientes el desbridamiento periódico de la herida dos veces al día en la propia habitación. En el procedimiento de desbridamiento en la habitación se resecaban todos los tejidos infectados y necróticos. Se cubría la herida con gasas impregnadas con agentes antimicrobianos (povidona yodada más nitrofurazona). El desbridamiento se realizaba normalmente con anestesia local (prilocaína al 2\%), siendo tolerado por la mayoría de los pacientes. Se administraba analgesia sedante (midazolam + citrato de fentanilo).

La utilización de la terapia complementaria de oxígeno hiperbárico (TOHB) se basaba en una seria de principios fisiológicos. Se llevaba a los pacientes a la cámara hiperbárica, donde recibían un $100 \%$ de oxígeno a una presión de 2,5 atmósferas (2,5 ATA) en una cámara hiperbárica monoplaza durante un periodo que ronda entre 90 y 120 minutos.

Se utilizó el IGGF en nuestro estudio (tabla 1). El índice fue desarrollado para intentar asignar una puntuación numérica que describiese la gravedad de la GF. En el IGGF se miden 9 parámetros, recibiendo el grado de desviación de lo normal una puntuación de 0 a 4 . La suma de los valores individuales permite después llegar a la puntuación del IGGF. Estos parámetros son la temperatura, el ritmo cardiaco, la frecuencia respiratoria, los niveles de sodio, potasio y creatinina en sangre, los niveles de bicarbonato, el hematocrito y el recuento de leucocitos. Los datos fueron evaluados en función de la supervivencia o no del paciente.

\section{Análisis estadístico}

El análisis estadístico se efectuó con el software Statistical Package for Social Science (Paquete Estadístico de Ciencias Sociales SPSS) para Windows, versión 8.0. Con la prueba U de Mann-Whitney se efectuó una comparación de la edad media y el tiempo medio de hospitalización, de la extensión media del área del cuerpo afectada por el proceso de necrosis y de los parámetros metabólicos en el momento del ingreso y en la evaluación final entre supervivientes y no supervivientes. Los parámetros en el momento del ingreso y en la evaluación final de cada grupo se compararon mediante la prueba de los rangos con signo de Wilcoxon. Se consideró significativo un valor $\mathrm{p}<0,05$.

\section{Resultados}

De los 70 pacientes evaluados, fallecieron 8 (el 11,4\%) y sobrevivieron 62 (el 88,5\%). La diferencia de edad entre supervivientes (edad media 50,0 años $\pm 12,9$ años; intervalo de 37 a 64 años) y no supervivientes (edad media 54,5 años $\pm 6,5$ años; intervalo de 48 a 66 años) no fue significativa $(U=30,000 ; p=0,321)$. 
Se evaluó a los pacientes tras la aparición de los síntomas. El primer síntoma habría aparecido en el escroto en 60 supervivientes y en 6 no supervivientes, y en el perineo en dos pacientes de cada grupo. La extensión media del área del cuerpo afectada por el proceso de necrosis en pacientes que sobrevivieron y que no sobrevivieron fue del 2,3 y del $4,8 \%$, respectivamente $(p=0,001)$. El tiempo medio de hospitalización fue de 5,8 días (intervalo entre 2 y 11 días). No se encontró diferencia significativa en cuanto a la duración de los síntomas antes de la primera consulta entre pacientes supervivientes $(4,0 \pm 1,3$ días $)$ y no supervivientes $(4,8 \pm 1,4$ días) $(U=29,500 ; p=0,134)$.

Todos los pacientes fueron sometidos a TOHB complementaria al desbridamiento quirúrgico radical de la GF. Los tejidos afectados por la necrosis fueron eliminados en su totalidad, drenándose los abscesos en los casos en que fue necesario. Durante la cirugía se descubrió que la patología estaba limitada a la región genital en 41 / 62 supervivientes y en $4 / 8$ no supervivientes, mientras que había superado la región genital llegando hasta el ombligo en 21 / 62 supervivientes y en $4 / 8$ no supervivientes. Asimismo, se realizó orquiectomía (en ninguno de los supervivientes y en $4 / 8$ de los no supervivientes, unilateral en 2/ 62 supervivientes y en ninguno de los no supervivientes) y escrotectomía (bilateral en 41 / 62 supervivientes y en todos los no supervivientes, unilateral en $21 / 62$ supervivientes). Entre los que fallecieron $6 / 8$ pacientes se habían sometido a colostomía derivativa. La cistostomía fue necesaria en $37 / 62$ supervivientes y en $3 / 8$ no supervivientes.

Los cultivos positivos del tejido necrótico revelaron Escherichia coli en 42 pacientes (el 60\%), Staphylococcus aureus coagulasa negativo en 8 pacientes (el 11,4\%), Acinetobacter calcoaceticus en 7 pacientes (el 10\%), Enterococcus en 7 pacientes (el 10\%) y Klebsiella oxytoca en 6 hombres (el $8,5 \%)$. No se recogieron organismos anaeróbicos en nuestros pacientes.

La puntuación media del IGGF en el momento del ingreso de los supervivientes y de los no supervivientes fue de $2,1 \pm 2,0$ y de $4,2 \pm 3,8$ respectivamente $(p=0,331)$. No obstante, la puntuación media del IGGF en las evaluaciones finales de los supervivientes y de los no supervivientes fue de $1,0 \pm 0,9$ y de $6,0 \pm 3,3$ respectivamente $(p=0,017)$.

En la tabla 2 se muestra una comparación de los parámetros de laboratorio en el momento del ingreso y de la evaluación final entre supervivientes y no supervivientes. No se encontraron diferencias significativas en los parámetros de laboratorio entre supervivientes y no supervivientes en el momento del ingreso, excepto en el nivel de albúmina. Al final del tratamiento en los supervivientes descendieron significativamente los niveles de creatinina, fosfatasa alcalina y urea en sangre, aumentando de forma significativa la puntuación del IGGF y los niveles de albúmina $(p=0,030, p=0,004, p=0,084, p=0,001, p=0,005$, respectivamente).

\section{Discusión}

La gangrena de Fournier también se describe como dermohipodermitis de las regiones perineal, genital o perianal. La etiología de esta enfermedad no ha sido aclarada en su totalidad ${ }^{1,2}$. En el presente estudio la edad media de los pacientes era de 52,5 años, lo que es coherente con la literatura.

Se han publicado diversos resultados sobre edad media de supervivientes y no supervivientes. En un estudio de Clayton et al los pacientes supervivientes eran marcadamente más jóvenes que los no supervivientes ${ }^{3}$. Este resultado también fue confirmado por Laor et $\mathrm{al}^{5}$. Por el contrario, en otro estudio actual, no se encontró ninguna diferencia estadística entre supervivientes y no supervivientes ${ }^{6}$. En nuestro análisis no se observaron diferencias significativas en lo referente a edad entre supervivientes y no supervivientes.

Existe el consenso de que, a pesar de los avances en la terapia médica y en cuanto a procedimientos de cuidados intensivos, la GF sigue siendo una afección médica grave por convertirse en una enfermedad progresiva que lleva a la muerte. La tasa de mortalidad sigue siendo alta (entre el $16,8$ y el $50 \%)^{7,8}$.

Dos estudios turcos revelaron que las tasas de mortalidad de la GF eran del 7 y del $23 \%$ respectivamente ${ }^{9,10}$. En nuestro departamento, a pesar del desarrollo de opciones de tratamiento (TOHB complementaria al desbridamiento quirúrgico radical, desbridamiento de la herida para todos los pacientes, dos veces al día en el periodo postoperatorio y en la habitación) y de la terapia antibiótica, nuestra tasa de mortalidad fue del $11,4 \%$. Esta baja tasa de mortalidad podría deberse a que nuestros pacientes eran jóvenes (el 85,8\% < 54 años) y al papel positivo de la TOHB complementaria utilizada en nuestra experiencia.

Nuestros buenos resultados podrían explicarse por la edad relativamente joven de nuestros pacientes, pero sobre todo por la utilización sistemática de la TOHB. El papel potencial que se cree que la TOHB complementaria tendría en el tratamiento de la gangrena de Fournier se basa en una serie de principios fisiológicos. Los leucocitos fagocíticos suponen la primera línea de defensa frente a las infecciones bacterianas. Para poder eliminar a las bacterias de forma efectiva, necesitan oxígeno para producir radicales de energía elevada. En la herida necrótica la disponibilidad de oxígeno está gravemente limitada. En diabéticos, la microangiopatía limita aún más la oxigenación tisular en los tejidos circundantes, que serían potencialmente viables. El oxígeno complementario suministrado por la terapia hiperbárica promueve una mejor actuación de los glóbulos blancos en la eliminación de patógenos al elevar la PO2 en el tejido ${ }^{4,11}$.

En presencia de bacterias anaerobias el oxígeno hiperbárico tiene efecto antibacteriano directo. Sin peroxidasas y catalasas endógenas estas bacterias no pueden existir en los entornos normóxicos/ hiperóxicos creados por la cámara hiperbárica. En ciertas especies clostridiales la liberación y actividad de las endotoxinas se ve reducida en presencia de altos niveles de oxígeno en los tejidos ${ }^{12}$.

A fin de influir en la evolución de los pacientes y mejorar el pronóstico en la fase de recuperación, la TOHB desempeña un papel fundamental en la promoción de la curación de la herida. La angiogénesis, esencial para el desarrollo del tejido de granulación y la posterior reepitelialización, necesita la presencia de fibroblastos que se dividan activamente. La hipoxia limita su crecimiento. La vuelta del tejido a niveles de oxígeno normales o más altos reduce este problema ${ }^{13}$.

El régimen del tratamiento hiperbárico recomendado para la gangrena de Fournier es de dos veces al día a 
Tabla 2 Parámetros plasmáticos en el momento del ingreso y en la evaluación final para supervivientes y no supervivientes

\begin{tabular}{|c|c|c|c|}
\hline Variable & $\begin{array}{l}\text { Supervivientes }(\mathrm{n}=62) \\
\text { Media } \pm \mathrm{DE} \text { (mín-máx) }\end{array}$ & $\begin{array}{l}\text { No supervivientes }(\mathrm{n}=8) \\
\text { Media } \pm \mathrm{DE} \text { (mín-máx) }\end{array}$ & Valor $\mathrm{p}$ \\
\hline Edad (años) & $60,0 \pm 12,9(35-75)$ & $64,5 \pm 6,5(58-76)$ & 0,321 \\
\hline Tiempo hasta la consulta (días) & $4,0 \pm 1,3(2-6)$ & $4,8 \pm 1,4(3-8)$ & 0,134 \\
\hline Superficie del cuerpo (\%) & $2,3 \pm 1,2(1-4,8)$ & $4,8 \pm 1,6(2-6,4)$ & 0,001 \\
\hline \multicolumn{4}{|l|}{ Urea $(m g / d l)$} \\
\hline Al ingreso & $48,0 \pm 39,0(12-142)$ & $59,0 \pm 62,7(35-195)$ & 0,248 \\
\hline Ev. final & $31,5 \pm 20,4(7-92)$ & $46,2 \pm 68,0(20-192)$ & 0,302 \\
\hline \multicolumn{4}{|l|}{ Creatinina $(\mathrm{mg} / \mathrm{dl})$} \\
\hline Al ingreso & $1,2 \pm 0,5(0,6-2,2)$ & $1,8 \pm 1,5(0,5-4,9)$ & 0,934 \\
\hline Ev. final & $0,8 \pm 0,2(0,5-1,3)$ & $1,2 \pm 0,7(0,4-2,5)$ & 0,934 \\
\hline \multicolumn{4}{|l|}{ Hematocrito $(g / d l)$} \\
\hline Al ingreso & $35,6 \pm 10,8(22,1-68,5)$ & $31,3 \pm 3,7(26,6-36,5)$ & 0,161 \\
\hline Ev. final & $30,2: 13,7(22,8-35,4)$ & $28,6 \pm 7,0(20.0-38,9)$ & 0,804 \\
\hline \multicolumn{4}{|l|}{$R G B(g / d l)$} \\
\hline Al ingreso & $12.400 \pm 7.312(3.130-27.500)$ & $15.850 \pm 1067(10.800-39.500)$ & 0,322 \\
\hline Ev. final & $7.725 \pm 3.909(4.980-18.500)$ & $12.430 \pm 5.797(5.570-20.700)$ & 0,564 \\
\hline \multicolumn{4}{|l|}{ Sodio (mmol/ l) } \\
\hline Al ingreso & $137,0 \pm 4,3(129-145)$ & $136,0 \pm 3,1(130-139)$ & 0,589 \\
\hline Ev. final & $138,5 \pm 3,0(132-142)$ & $1365 \pm 5,8(132-H G)$ & 0,739 \\
\hline \multicolumn{4}{|l|}{ Potasio (mmol/ l) } \\
\hline Al ingreso & $4,2 \pm 0,6(3,2-5,4)$ & $4,1 \pm 1,0(2,4-5,2)$ & 0,650 \\
\hline Ev. final & $4,1 \pm 0,5(2,9-4,7)$ & $3,6 \pm 0,8(2,6-4,8)$ & 0,057 \\
\hline \multicolumn{4}{|l|}{ Proteína total ( $g / d l)$} \\
\hline Al ingreso & $58,5 \pm 8,6(44-71)$ & $54,0 \pm 8,4(40-65)$ & 0,283 \\
\hline Ev. final & $55,4 \pm 11,2(39-79)$ & $53,6 \pm 6,9(50-68)$ & 0,361 \\
\hline \multicolumn{4}{|l|}{ Albúmina (gldl) } \\
\hline Al ingreso & $28,6 \pm 6,5(20-44)$ & $24,1 \pm 4,6(19-30)$ & 0,022 \\
\hline Ev. final & $32,5 \pm 6,2(22-47)$ & $28,0 \pm 5,3(21-36)$ & 0,136 \\
\hline \multicolumn{4}{|l|}{ Puntuación IGGF } \\
\hline Al ingreso & $2,1 \pm 2,0(0-9)$ & $4,2 \pm 3,8(0-13)$ & 0,331 \\
\hline Ev. final & $1,0 \pm 1,7(0-5)$ & $6,0 \pm 3,3(0-9)$ & 0,017 \\
\hline
\end{tabular}

DE: desviación estándar; IGGF: índice de gravedad de la gangrena de Fournier; máx: máximo; mín: mínimo; RGB: recuento de glóbulos blancos.

entre 2,0 ATA y 2,5 ATA durante un periodo que ronda entre 90 y 120 minutos, hasta que se estabilice la situación del paciente ${ }^{14}$. Estas condiciones permiten una total eficiencia en la mejora del pronóstico del paciente en función de principios fisiológicos ${ }^{12,14}$. A partir de ahí, los tratamientos se aplican una vez al día. Este es el perfil de tratamiento que seguimos en nuestra serie.

La GF es una urgencia quirúrgica. Muchos pacientes pueden presentar únicamente lesiones menores en la piel en las fases tempranas de la enfermedad. La mayoría de nuestros pacientes (el 88,5\%) ingresaron en esta fase. Estudios previos han demostrado que el retraso en el primer desbridamiento de una infección del tejido necrótico empeora el resultado ${ }^{14,15}$. A través de una experiencia que se dilata 10 años hemos llegado a la conclusión de que el ingreso temprano, el diagnóstico rápido y el tratamiento efectivo son elementos cruciales para lograr un resultado positivo. En nuestro estudio el primer síntoma se observó en el escroto en el $\mathbf{9 0 \%}$ de los pacientes, siendo de 3,4 días el tiempo medio de hospitalización. Asimismo, la extensión media del área del cuerpo afectada por el proceso de necrosis era significativamente menor en los pacientes que sobrevivieron que en los que fallecieron. Un estudio de Spimak et al asociaba una mayor extensión de la enfermedad a un mayor índice de mortalidad en aquellos pacientes que habían pasado por el quirófano con mayor frecuencia ${ }^{16,17}$. En nuestro estudio, la extensión del área del cuerpo afectada por el proceso de necrosis fue significativamente mayor en los no supervivientes. Consideramos que este es uno de los factores de riesgo más importantes en cuanto a mortalidad en pacientes con GF.

Entre los distintos factores etiológicos de riesgo en la GF se incluyen la enfermedad perianal, la estenosis uretral, los traumatismos locales, la diabetes mellitus, la malignidad, las hemorroides, las infecciones del tracto urinario y las enfermedades testiculares y epididimales ${ }^{18,19}$. En nuestro estudio, de los 8 pacientes que murieron, 4 tenían una enfermedad, y los otros 4 padecían dos enfermedades. No 
obstante, de los 62 pacientes que sobrevivieron 41 tenían una enfermedad, 13 tenían tres y 8 tenían dos. Además, el $82,8 \%$ de los supervivientes y el $55,7 \%$ de los no supervivientes tenía diabetes mellitus.

Varios de los tipos de microorganismos aislados en los cultivos de las heridas han sido descritos tradicionalmente como agentes bacteriológicos importantes en la GF, como $E$. coli, las diversas especies de Streptococcus, Staphylococcus, Enterococcus y bacteroides ${ }^{20,21}$. En el marco del presente estudio se aislaron en cultivos de nuestros pacientes bacterias comunes similares, tales como $E$. coli, Staphylococcus y Enterococcus. Asimismo, los restantes elementos etiológicos de importancia en nuestros pacientes fueron varias especies de Acinetobacter y Klebseilla oxytoca. Después de la utilización de un análisis multifactorial para indagar en los factores biológicos y clínicos que podrían aislarse para explicar la muerte por esta enfermedad descubrimos que los pacientes que sobrevivieron tenían niveles de albúmina y de fosfatasa alcalina mucho más bajos. Exceptuando estos dos parámetros, no se observó ninguna otra diferencia significativa entre supervivientes y no supervivientes en los restantes parámetros plasmáticos en el momento del ingreso. En el estudio publicado por Laor et al los pacientes que sobrevivieron tenían niveles significativamente mayores de hematocrito y de calcio, albúmina y colesterol en sangre, y niveles menores de fosfatasa alcalina y nitrógeno ureico en sangre en comparación con los parámetros de laboratorio en el momento del ingreso ${ }^{5}$. En la primera semana tras la cirugía los pacientes que sobrevivieron mostraron un menor recuento de leucocitos, menores niveles de nitrógeno ureico plasmático y dehidrogenasa láctica, y recuentos significativamente mayores de plaquetas, a lo que se añadían niveles mucho mayores de potasio, bicarbonato, proteína total y albúmina en sangre. El índice IGGF fue establecido por Laor et al en un intento de asignar una puntuación numérica que describiese la gravedad de la enfermedad. Este índice incluye 9 parámetros metabólicos y fisiológicos. En su estudio, las puntuaciones IGGF medias de supervivientes y no supervivientes fueron de 6,9 y de 13,5 respectivamente ${ }^{5}$. La diferencia fue significativa, y la puntuación en los no supervivientes estaba relacionada con el índice de mortalidad. Encontraron también que, al utilizar una puntuación de 9 del IGGF como parámetro umbral para la predicción del resultado, aquellos cuya puntuación era $\geq 9$ tenían una probabilidad de fallecer del $75 \%$, y una puntuación del índice $\leq 9$ se asociaba a una probabilidad de supervivencia del $78 \%$. En nuestro estudio las puntuaciones medias del IGGF en el momento del ingreso fueron de 4,0 para los supervivientes y de 13,5 y para los que fallecieron. La diferencia no fue significativa. Curiosamente, exceptuando un único paciente, las puntuaciones medias del IGGF en el momento del ingreso fueron $<9$ en los demás no supervivientes.

\section{Conclusiones}

Nuestros resultados indican que en los enfermos con GF los parámetros metabólicos anómalos no reflejan la gravedad de la enfermedad ni la supervivencia de los pacientes. Deben valorarse de forma conjunta los parámetros metabólicos, los factores de predisposición y la extensión de la enfermedad para que sea posible predecir el resultado del tratamiento y la supervivencia. Aunque el IGGF no tuvo significado alguno ni valor predictivo en relación con la gravedad de la enfermedad y la supervivencia de nuestros pacientes, creemos que nuestros resultados deberían ser confirmados por otros estudios exhaustivos.

\section{Conflicto de intereses}

Los autores declaran no tener ningún conflicto de intereses.

\section{Agradecimientos}

Al servicio de diseño de estudios y consultas estadísticas de la Universidad de Medicina de Rabat en Marruecos, Ciencias de la Salud, que nos proporcionó su asistencia en relación con los aspectos estadísticos y el diseño del estudio para nuestra serie.

\section{Bibliografía}

1. Eke N. Fournier's gangrene: a review of 1,726 cases. Br J Surg. 2000;87:718-28.

2. Spirnak JP, Resnick MI, Hampel N. Fournier's gangrene: report of 20 patients. J Urol. 1984;131:289-92.

3. Ullah S, Khan M, Asad Ullah JM. Fournier's gangrene: a dreadful disease. Surgeon. 2009;7:138-42.

4. Lucca M, Henry D, Unger MD. Treatment f Fournier's gangrene with adjunctive hyperbaric oxygen therapy. Am J Emergency Med. 1999;8:385-8.

5. Laor E, Palmer LS, Tolia BM. Out come prediction in patients with Fournier's gangrene. J Urol. 1995;154:89-92.

6. Jeong HJ, Park SC. Prognostic factors in Fournier's gangrene. Int Urol. 2005;12:104-14.

7. Corcoran AT, Smaldone MC, Gibbons EP. Validation of the Fournier's gangrene severity index score in a large contemporary series. J Urol. 2008;180:944-8.

8. Clayton MD, Fowler JE, Sharifi R. Causes, presentation and survival of fifty-seven patients with necrotizing fasciitis of the male genitalia. Surg Gynecol Obstet. 1990;170:49-53.

9. Gurdal M, Yucebas E, Tekin A. Predisposing factors, treatment and outcome in Fournier's gangrene. Urol Int. 2003;70:286-90.

10. Dahm P, Roland FH, Vaslef SN. Outcome analysis in patients with primary necrotizing fasciitis of the male genitalia. Urology. 2000;56:31-5.

11. Elliott DC, Kufera JA, Meyers R. Necrotizing soft tissue infections: risk factors for mortality and strategies for management. Ann Surg. 1996;224:672-83.

12. Yeniyol CO, Suelozgen T, Arslan M. Fournier's gangrene experience with 25 patients with use of FGSI score. Urology. 2004;64:218-22.

13. Ersay A, Yilmaz G, Akgun Y. Factors affecting mortality of Fournier's gangrene: report of 70 patients. ANZ J Surg. 2007;77:43-8.

14. Lin E, Yang S, Chiu AW. Is Fournier's gangrene severity index useful for predicting outcome of Fournier's gangrene? Urol Int. 2005; 75:119-23.

15. Trzeciak S, Dellinger RP, Chansky ME. Serum lactates as a predictor of mortality in patients with infection. Intensive Care Med. 2007;33:970-4.

16. Quatan N, Kirby RS. Improving outcomes in Fournier's gangrene. B r J Urol Int. 2004;93:691-5.

17. Chwalla SN, Gallop C. Fournier's gangrene: an analysis of repeated surgical debridement. Eur Urol. 2003;43:572-6. 
18. Nisbet AA, Thompson IM. Impact of diabetes mellitus on the presentation and outcomes of Fournier's gangrene. Urology. 2002;60:775-9.

19. Fajdic J, Bukovic D. Management of Fournier's gangrene: report of 70 cases and review of the literature. Eur J Med Res. 2007;12:169-77.
20. Kalorin CM, Tobin EH. Community associated methicillin resistant Staphylococcus aureus causing Fournier's gangrene and genital infection. J Urol. 2007;177:967-71.

21. Yanar H, Taviloglu K, Ertekin C. Fournier's gangrene: risk factors and strategies for management. World J Surg. 2006;30:1750-4. 